\title{
INFINITELY MANY TRAVELING WAVE SOLUTIONS OF A GRADIENT SYSTEM
}

\author{
DAVID TERMAN
}

\begin{abstract}
We consider a system of equations of the form $u_{t}=u_{x x}+\nabla F(u)$. A traveling wave solution of this system is one of the form $u(x, t)=U(z), z=x+\theta t$. Sufficient conditions on $F(u)$ are given to guarantee the existence of infinitely many traveling wave solutions.
\end{abstract}

\section{Introduction.}

A. Statement of the problem. Consider the system of reaction-diffusion equations

$$
u_{1 t}=u_{1 x x}+f_{1}\left(u_{1}, u_{2}\right), \quad u_{2 t}=u_{2 x x}+f_{2}\left(u_{1}, u_{2}\right)
$$

where $u_{1}$ and $u_{2}$ are functions of $(x, t) \in \mathbf{R} \times \mathbf{R}^{+}$. We assume that $f_{1}$ and $f_{2}$ are derived from some potential. That is, there exists a function $F \in C^{2}\left(\mathbf{R}^{2}\right)$ such that

$$
f_{i}\left(u_{1}, u_{2}\right)=\frac{\partial F}{\partial u_{i}}\left(u_{1}, u_{2}\right), \quad i=1,2,
$$

for each $\left(u_{1}, u_{2}\right) \in \mathbf{R}^{2}$. By a traveling wave solution of (1A.1) we mean a nonconstant, bounded solution of the form

$$
\left(u_{1}(x, t), u_{2}(x, t)\right)=\left(U_{1}(z), U_{2}(z)\right), \quad z=x+\theta t
$$

A traveling wave solution corresponds to a solution which appears to be traveling with constant shape and velocity. Our goal is to prove that for a certain class of potentials, there exists infinitely many traveling wave solutions of (1A.1).

We shall assume that the graph of $F(U)$ is as shown in Figure 1. Precise assumptions of $F$ will be given shortly. For now we assume that $F$ has at least three local maxima. These are at $\left(U_{1}, U_{2}\right)=A, B$ and $C$ with $F(A)<F(B)<F(C)$. We will be interested in traveling wave solutions which satisfy

$$
\lim _{z \rightarrow-\infty}\left(U_{1}(z), U_{2}(z)\right)=A \text { and } \lim _{z \rightarrow-\infty}\left(U_{1}(z), U_{2}(z)\right)=B
$$

Motivation for studying this problem will be given shortly.

Received by the editors January 16, 1986 and, in revised form, May 15, 1986.

1980 Mathematics Subject Classification (1985 Revision). Primary 35B35, 35K55; Secondary 35 B40.

Supported in part by the National Science Foundation under grant \# DMS 8401719. 


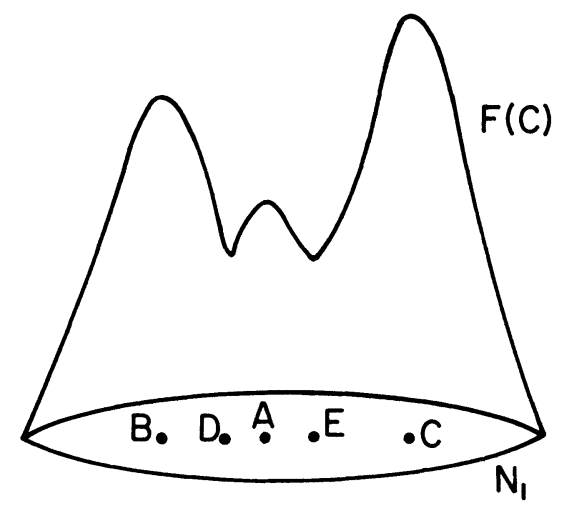

FIGURE 1

If $\left(U_{1}(z), U_{2}(z)\right)$ is a traveling wave solution of (1A.1) and $V_{i}(z)=U_{i}^{\prime}(z)$, $i=1,2$, then $\left(U_{1}, U_{2}, V_{1}, V_{2}\right)$ satisfies the first order system of ordinary differential equations

$$
\begin{array}{ll}
U_{1}^{\prime}=V_{1}, & V_{1}^{\prime}=\theta V_{1}-F_{u_{1}}\left(U_{1}, U_{2}\right), \\
U_{2}^{\prime}=V_{2}, & V_{2}^{\prime}=\theta V_{2}-F_{u_{2}}\left(U, U_{2}\right)
\end{array}
$$

together with the boundary conditions

$$
\begin{aligned}
& \text { (a) } \lim _{z \rightarrow-\infty}\left(U_{1}(z), U_{2}(z), V_{1}(z), V_{2}(z)\right)=(A, \mathcal{O}), \\
& \text { (b) } \lim _{z \rightarrow+\infty}\left(U_{1}(z), U_{2}(z), V_{1}(z), V_{2}(z)\right)=(B, \mathcal{O}) .
\end{aligned}
$$

Here we have set $\mathcal{O}=(0,0)$. For convenience we let $U=\left(U_{1}, U_{2}\right)$ and $V=\left(V_{1}, V_{2}\right)$.

Our goal is to prove that for certain assumptions on $F$ there exist infinitely many $\theta$ for which there exists a solution of (1A.5), (1A.6).

B. Assumptions on $F$. We assume that the graph of $F(U)$ is as shown in Figure 1. The precise assumptions on $F$ are as follows:

(F1) $F \in C^{2}\left(\mathbf{R}^{2}\right)$.

(F2) $F$ has at least three nondegenerate local maxima. These are at $A=\left(A_{1}, A_{2}\right)$, $B=\left(B_{1}, B_{2}\right)$, and $C=\left(C_{1}, C_{2}\right) . F$ has at least two saddles at $D=\left(D_{1}, D_{2}\right)$ and $E=\left(E_{1}, E_{2}\right)$.

(F3) $F(A)<F(B)<F(C)$ and $B_{1}<D_{1}<A_{1}<E_{1}<C_{1}$. Moreover there exists an $\alpha_{0}$ such that if $\alpha$ is any critical point of $F$ with $\alpha \notin\{A, B, C\}$, then $F(\alpha)<$ $F(A)-\alpha_{0}$.

(F4) There exists $W$ such that if $K<W$, then $\{U: F(U) \geqslant K\}$ is convex.

(F5) If $U_{1}=D_{1}$ or $E_{1}$, then $\left(\partial F / \partial U_{1}\right)\left(U_{1}, U_{2}\right)=0$ for all $U_{2} \in \mathbf{R}$.

(F6) Let

$$
\begin{aligned}
& N_{1}=\{U: F(U) \geqslant W\}, \\
& X_{1}=\left\{U \in N_{1}: U_{1}<D_{1}\right\}, \\
& X_{2}=\left\{U \in N_{1}: D_{1}<U_{1}<E_{1}\right\}, \\
& X_{3}=\left\{U \in N_{1}: E_{1}<U_{1}\right\} .
\end{aligned}
$$


Suppose that $(U(z), V(z))$ is a bounded solution of (1A.5) with $\theta=0$ which satisfies the following for $i=1,2$, or 3,

(a) $U(z) \in X_{i}$ for all $z \in \mathbf{R}$,

(b) $F(U(z))>F(A)-\alpha_{0}$ for some $z \in \mathbf{R}$,

where $\alpha_{0}$ was defined in (F3). Then $U(z)$ is identically equal to one of the critical points $A, B$, or $C$, and $V(z)=\mathcal{O}$ for all $z \in \mathbf{R}$.

Remarks concerning these assumptions are certainly in order. These remarks are given in $\$ 1 \mathrm{E}$. First, we state our main result and then try to motivate the results and assumptions on $F$ by briefly discussing the scalar equation $u_{t}=u_{x x}+g(u)$.

C. The main result.

THEOREM 1. Assume that $f_{1}$ and $f_{2}$ satisfy (1A.2) and $F\left(U_{1}, U_{2}\right)$ satisfies (F1) to (F6). Then there exists infinitely many traveling wave solutions of (1A.1) which satisfy (1A.4).

REMARK. By infinitely many traveling waves we mean that there exist infinitely many values of $\theta$ for which there exists a solution of (1A.5), (1A.6).

D. The scalar equation. This paper is a generalization of previous work on the scalar equation

$$
u_{t}=u_{x x}+g(u)
$$

In [3], a rather complete description of which waves exist is given for general nonlinearities $g(u)$. In [3], we used graph theory to prove the existence of traveling waves. In this paper we demonstrate what takes the place of the directed graphs and arrays of integers, which were used in [3], for higher dimensional gradient systems.

E. Remarks on the assumptions on $F$.

REMARK 1. (F4) will be used to prove that the set of bounded solutions of (1A.5) is compact.

REMARK 2. (F6) guarantees that the set of bounded solutions of (1A.5) is not too bizarre. One may think of (1A.5) with $\theta=0$ as describing the motion of a ball rolling along the landscape defined by the graph of $F$. There may exist bounded solutions of (1A.5) with $\theta=0$, because the ball may roll back and forth between the mountain peaks given by $F(A), F(B)$, and $F(C)$. Assumption (F6) implies that these are the only bounded solutions, besides the critical points, which lie above $F(A)-\alpha_{0}$. This condition will be satisfied if the level sets of $F(U)$ are sufficiently nice (some starlike condition, for example). It is not clear whether or not this condition is necessary to guarantee the existence of infinitely many traveling wave solutions.

REMARK 3. (F5) is perhaps the most unreasonable assumption. It can be weakened slightly as follows. Let

$$
l_{D}=\left\{U \in N_{1}: U_{1}=D_{1}\right\}, \quad l_{E}=\left\{U \in N_{1}: U_{1}=E_{1}\right\} .
$$

Then (F5) implies that if $U \in l_{D} \cap N_{1}$ or $U \in l_{E} \cap N_{1}$, then $\nabla F(U)$ is tangent to $l_{D}$ or $l_{E}$, respectively. Our result remains true if this property holds for some lines $l_{D}$ and $l_{E}$ through $D$ and $E$, not necessarily the ones given in (1E.1). We choose $l_{D}$ and $l_{E}$ as in (1E.1) only for convenience. We do feel that our method of proof should 
carry over to a more general assumption than (F5). In [4, Appendix 5] we describe how one should be able to weaken (F5).

F. Description of the proof. The proof of Theorem 1 is quite geometrical. The purpose of this subsection is to introduce the basic geometrical features of the proof. Note that each solution of (1A.5) corresponds to a trajectory in four-dimensional phase space. The boundary conditions (1A.6) imply that we are looking for a value of $\theta$ for which there exists a trajectory in phase space which approaches the equilibrium $(A, \mathcal{O})$ as $z \rightarrow-\infty$ and the equilibrium $(B, \mathcal{O})$ as $z \rightarrow+\infty$. Hence, we are looking for a trajectory which lies in both $W_{A}$, the unstable manifold at $(A, \mathcal{O})$, and $W_{B}^{s}$, the stable manifold at $(B, \mathcal{O})$.

The first step in the proof of Theorem 1 is to obtain apriori bounds on the bounded solutions of (1A.5). This is done in $\S 2$. We prove that there exists a $T$ such that no solutions of (1A.5), (1A.6) exist for $\theta>T$. We also construct a four-dimensional box, $N$, which contains all the bounded solutions of (1A.5).

We then construct a subset $\mathscr{E}$ of the boundary of $N$ with the property that each nontrivial trajectory in $W_{A}$, for $\theta<T$, can only leave $N$ through $\mathscr{E}$. The most interesting feature of $\mathscr{E}$ is that it has four topological holes.

Now choose $\theta_{0}$ so that no solutions of (1A.5), (1A.6) exist for $\theta=\theta_{0}$. We prove that each nontrivial trajectory in $W_{A}$ must leave $N$. Because the dimension of $W_{A}$ is two, the places where $W_{A}$ leaves $N$ define a curve, $\Lambda\left(\theta_{0}\right)$, in $\mathscr{E}$. We define an algebraic object, $\Gamma\left(\theta_{0}\right)$, which describes how $\Lambda\left(\theta_{0}\right)$ winds around the four holes in $\mathscr{E}$. $\Gamma\left(\theta_{0}\right)$ will be an element of $F_{4}$, the free group on four elements. The definition of $\Gamma\left(\theta_{0}\right)$ is given in $\S 4 \mathrm{~B}$. In $\S 4 \mathrm{~B}$ we set things up a bit more generally than described here in anticipation of future papers in which we characterize the solutions of (1A.5), (1A.6) by how many times they wind around in phase space. This notion of winding number will play an important role in this paper, as we describe shortly. The winding number is defined precisely in $\S 3 \mathrm{~B}$.

The algebraic invariant, $\Gamma\left(\theta_{0}\right)$, will have the following important property:

Proposition A (See Proposition 4B.2). Suppose that $\theta_{0}<\theta_{1}$ are chosen so that there exist no solutions of (1A.5), (1A.6) for $\theta \in\left[\theta_{0}, \theta_{1}\right]$. Then $\Gamma\left(\theta_{0}\right)=\Gamma\left(\theta_{1}\right)$.

The next step in the proof of Theorem 1 is to assign to each element $\Gamma \in F_{4}$ a positive integer $|\Gamma|$. We prove

Proposition B (SeE Proposition 5A.1). Let $M$ be a positive integer. There exists $\theta_{M}$ such that if $\theta_{0}<\theta_{M}$ and no solutions of (1A.5), (1A.6) exist for $\theta=\theta_{0}$, then $\left|\Gamma\left(\theta_{0}\right)\right|>M$.

Theorem 1 then follows from Proposition A and Proposition B.

The key steps in the proof of Proposition B are Proposition 3C.1 and Proposition 4C.1. In Proposition 4C.1 we give a relationship between $\left|\Gamma\left(\theta_{0}\right)\right|$ and the winding number of trajectories in $W_{A}$ for $\theta=\theta_{0}$. In Proposition 3C.1 we prove that if $\theta_{0}$ is very small then there must exist a trajectory in $W_{A}$, for $\theta=\theta_{0}$, with large winding number. 


\section{The isolating neighborhood.}

A. Basic definitions and a preliminary result. Recall the set $N_{1}$ defined in (1B.1). Let

$$
N_{2}=\left\{(U, V): U \in N_{1},\|V\| \leqslant \bar{V}\right\}
$$

where $\bar{V}$ is a large number to be determined. Let

$$
\begin{aligned}
P_{D} & =\left\{(U, V): U_{1}=D_{1} \text { and } V_{1}=0\right\}, \\
P_{E} & =\left\{(U, V): U_{1}=E_{1} \text { and } V_{1}=0\right\}, \\
N & =N_{2} \backslash\left(P_{D} \cup P_{E}\right) .
\end{aligned}
$$

REMARK. $N$ is topologically a four-dimensional box with two holes, $P_{D}$ and $P_{E}$, removed. This is topologically equivalent to a two-dimensional disc with two points removed.

We first prove

LEMMA 2A.1. For all $\theta, P_{D}$ and $P_{E}$ are invariant with respect to the flow defined by (1A.5).

Proof. We wish to show that if $\left(U\left(z_{0}\right), V\left(z_{0}\right)\right) \in P_{D}$ or $P_{E}$ for some $z_{0}$, then $(U(z), V(z)) \in P_{D}$ or $P_{E}$ for all $z$. However, from (1A.5) and (F5) we conclude that on $P_{D}$ or $P_{E}$,

$$
U_{1}^{\prime}=V_{1}=0
$$

and

$$
V_{1}^{\prime}=\theta V_{1}-F_{u_{1}}\left(u_{1}, u_{2}\right)=0
$$

These two equalities prove the lemma. An immediate consequence of the lemma is

Corollary 2A.2. For each $\theta$, the unstable manifold at $(A, \mathcal{O})$ does not intersect $P_{D}$ or $P_{E}$.

We wish to prove that all bounded solutions of (1A.4) lie in $N_{2}$ if $\bar{V}$ is sufficiently large. Together with the corollary, this will imply that solutions of (1A.5), (1A.6) must lie in $N$. We begin with

LEMMA 2A.3. The projection onto $U$-space of every bounded solution of (1A.5) lies in $N_{1}$. Moreover, there cannot exist a solution of (1A.5) whose projection onto U-space is internally tangent to $\partial N_{1}$, the boundary of $N_{1}$.

Proof. The proof follows that given in Conley [1]. Choose $K \leqslant W$ and suppose that $(U(z), V(z))$ is a solution of (1A.5) which satisfies for some $z_{0}, F\left(U\left(z_{0}\right)\right)=K$, and

$$
\left.\frac{\partial F}{\partial z}\right|_{z_{0}}=\left\langle\nabla F\left(U\left(z_{0}\right)\right), V\left(z_{0}\right)\right\rangle=0 .
$$


Then

$$
\begin{aligned}
\left.\frac{d^{2} F}{d z^{2}}\right|_{z_{0}}= & d^{2} F(U)+\theta\left\langle\nabla F\left(U\left(z_{0}\right)\right), V\left(z_{0}\right)\right\rangle \\
& -\left\langle\nabla F\left(U\left(z_{0}\right)\right), \nabla F\left(U\left(z_{0}\right)\right)\right\rangle<0
\end{aligned}
$$

since the assumption that the level set $\{F(V) \geqslant K\}$ is convex implies that

$$
d^{2} F(\xi)<0 \text { if } \xi \neq 0 \text { and }\langle\nabla F, \xi\rangle=0 .
$$

This implies that there cannot exist any internal tangencies on the level set $\{F(U)=K\}$ for all $K \leqslant W$.

On any solution which leaves the set where $F(U) \geqslant W$ there is a point where $F<W$ and either $d F / d z<0$ or $d F / d z>0$. Suppose that $F\left(U\left(z_{0}\right)\right)<W$ and $d F /\left.d z\right|_{z_{0}}<0$. Then (2A.2) implies that $F(U(z))$ is strictly decreasing for $z \geqslant z_{0}$. Therefore, if the solution is bounded, it would have to go to a rest point in the set where $F<W$. Since there are no rest points, the solution must be unbounded in forward time. A similar argument shows that if $F\left(U\left(z_{0}\right)\right)<W$ and $d F /\left.d z\right|_{z_{0}}>0$, then the solution is unbounded in backward time.

REMARK. The proof of this last result shows that if $U(z)$ leaves $N_{1}$ in forward or backward time, then it can never return to $N_{1}$.

B. The energy $H$. Consider the function $H(U, V)=\frac{1}{2}\langle V, V\rangle^{2}+F(U)$ where $\langle V, V\rangle$ is the usual inner product in $\mathbf{R}^{2}$. If $(U(z), V(z))$ is a solution of (1A.5) we sometimes write $H(z)=H(U(z), V(z))$. An important fact is that on solutions of (1A.5),

$$
H^{\prime}(z)=\theta\langle V(z), V(z)\rangle^{2}
$$

Therefore, if $\theta \neq 0$, then

$$
H^{\prime}(z)>0
$$

and $H(z)$ is increasing on solutions of (1A.5). An immediate consequence of this is

Proposition 2B.1. The only bounded solutions of (1A.5) with $\theta>0$ are critical points or trajectories which connect critical points.

Note that if $(U(z), V(z))$ is a solution of (1A.5), (1A.6), then

$$
\lim _{z \rightarrow-\infty} H(z)=F(A) \text { and } \lim _{z \rightarrow+\infty} H(z)=F(B) .
$$

C. $A$ bound on $\theta$. In this section we prove

LEMMA 2C.1. There exists $T$ such that no solutions of (1A.5), (1A.6) exist with $\theta \geqslant T$.

Proof. For $\lambda>0$, let

$$
S_{\lambda}=\left\{(U, V):\left|V_{1}\right| \geqslant \lambda\left|U_{1}-A_{1}\right| \text { and }\left|V_{2}\right| \geqslant \lambda\left|U_{2}-A_{2}\right|\right\} .
$$

We prove that given $\lambda$ there exists $T_{\lambda}$ such that if $\theta \geqslant T_{\lambda}, W_{A^{\theta}}$ is the unstable manifold of (1A.5) at $(A, \mathcal{O})$, and $(U(z), V(z)) \in W_{A^{\theta}}$, then $(U(z), V(z)) \in S_{\lambda}$. Because every solution of (1A.5), (1A.6) must lie in $W_{A^{\theta}}$, and $(B, \mathcal{O}) \notin S_{\lambda}$, for any $\lambda>0$, this will imply the desired result. 
We first prove that there exists $T_{\lambda}$ such that if $\theta \geqslant T_{\lambda}$, then $S_{\lambda}$ is positively invariant for the flow (1A.5). To prove this we show that on the boundary of $S_{\lambda}$, the vector field given by the right side of (1A.5) points into $S_{\lambda}$.

There are many cases to consider. Suppose, for example, that $V_{1}=\lambda\left(U_{1}-A_{1}\right)$, $U_{1}>A_{1}$, and $\left|V_{2}\right| \geqslant \lambda\left|U_{2}-A_{2}\right|$. Let $n=(\lambda,-1)$ be a vector outwardly normal to $\left\{\left(U_{1}, V_{1}\right): V_{1}=\lambda\left(U_{1}-A_{1}\right)\right\}$. Then

$$
\begin{aligned}
n \cdot\left(U_{1}^{\prime}, V_{1}^{\prime}\right) & =\lambda V_{1}-\theta V_{1}+F_{u_{1}}(U) \\
& =(\lambda-\theta)\left(U_{1}-A_{1}\right)+F_{u_{1}}(U)<0
\end{aligned}
$$

for $\theta$ sufficiently large. A similar proof works in the other cases.

To complete the proof of the lemma we show that $T_{\lambda}$ can be chosen so that if $\theta \geqslant T_{\lambda}$ and $(U(z), V(z)) \in W_{A^{\theta}}$, then there exists $z_{0}$ such that $(U(z), V(z)) \in S_{\lambda}$ for $z<z_{0}$. This is proved by linearizing $(1 \mathrm{~A} .5)$ at $(A, \mathcal{O})$ and showing that the positive eigenvectors point into $S_{\lambda}$.

If we set $A^{\prime}=(A, \mathcal{O})$ and

$$
\left.\frac{\partial^{2} F}{\partial u_{1}^{2}}\right|_{A^{\prime}}=a,\left.\quad \frac{\partial^{2} F}{\partial u_{1} \partial u_{2}}\right|_{A^{\prime}}=b,\left.\quad \frac{\partial^{2} F}{\partial u_{2}^{2}}\right|_{A^{\prime}}=c,
$$

then the linear system at $A^{\prime}$ is

$$
\left[\begin{array}{l}
U_{1} \\
U_{2} \\
V_{1} \\
V_{2}
\end{array}\right]^{\prime}=\left[\begin{array}{cccc}
0 & 0 & 1 & 0 \\
0 & 0 & 0 & 1 \\
-a & -b & \theta & 0 \\
-b & -c & 0 & \theta
\end{array}\right]\left[\begin{array}{c}
U_{1} \\
U_{2} \\
V_{1} \\
V_{2}
\end{array}\right] .
$$

To compute the eigenvalues and eigenvectors of this system let

$$
M=\left[\begin{array}{ll}
a & b \\
b & c
\end{array}\right]
$$

be the Hessian matrix of $F$ at $A$. Since $F(U)$ obtains a local maximum at $A$ it follows that $M$ has negative eigenvalues, which we denote by $-\lambda_{1}$ and $-\lambda_{2}$. The eigenvalues of (2C.1) are then $\sigma_{1}^{+}, \sigma_{1}^{-}, \sigma_{2}^{+}, \sigma_{2}^{-}$where for $i=1,2, \sigma_{i}^{+}$and $\sigma_{i}^{-}$are roots of the polynomial $\sigma^{2}-\sigma \theta-\lambda_{i}=0$. Since this polynomial has one positive and one negative root we may assume that

$$
\sigma_{1}^{-}<0<\sigma_{1}^{+} \text {and } \sigma_{2}^{-}<0<\sigma_{2}^{+} .
$$

Let $W_{1}$ and $W_{2}$ be eigenvectors of $M$ corresponding to $-\lambda_{1}$ and $-\lambda_{2}$, respectively. Then eigenvectors corresponding to $\sigma_{1}^{ \pm}$and $\sigma_{2}^{ \pm}$are then

$$
p_{i}^{ \pm}=\left(w_{i}, \sigma_{i}^{ \pm} w_{i}\right), \quad i=1,2 .
$$

Because $\sigma_{i}^{+} \rightarrow \infty, i=1,2$, as $\theta \rightarrow \infty$ it follows that $p_{1}^{+}$and $p_{2}^{+}$point into $S_{\lambda}$ if $\theta$ is sufficiently large. Because $W_{A^{\theta}}$ is tangent to the linear space spanned by $p_{1}^{+}$and $p_{2}^{+}$ this implies the desired result. 
REMARK. Our proof proved more than Lemma 2C.1. We also proved

Corollary 2C.2. Fix $\delta>0$ and let $A_{\delta}=\{U:\|U-A\|=\delta\}$. Given $\lambda>0$ there exists $M$ such that if $\theta>M,(U(z), V(z)) \in W_{A^{\theta}}, U\left(z_{0}\right) \in A_{\delta}$, and $\|U(z)-A\|<\delta$ for $z<z_{0}$, then $\left\|V\left(z_{0}\right)\right\|>\lambda$.

Proof. Let $\lambda_{1}=2 \lambda / \delta$ and $M=T_{\lambda_{1}}$, as in the previous lemma. If $\theta>M$, $(U(z), V(z)) \in W_{A^{\theta}}$, and $\|U(z)-A\|=\delta$. Then either $\left|U_{1}-A_{1}\right|>\delta / 2$ or $\left|U_{2}-A_{2}\right|$ $>\delta / 2$. From the previous lemma, either $\left\|V_{1}\right\| \geqslant \lambda_{1} \delta / 2=\lambda$ or $\left\|V_{2}\right\| \geqslant \lambda_{1} \delta / 2=\lambda$.

D. $A$ bound on $V$. Recall that in the definition of $N$ we needed a constant $\bar{V}$ which has not been defined yet. $\bar{V}$ will serve as an a priori bound on $\|V\|$ of solutions of (1A.5), (1A.6). That such a bound exists follows from

Proposition 2D.1. Assume that $\theta<T . \bar{V}$ can be chosen so that if $U\left(z_{0}\right) \in N_{1}$ and $\left\|V\left(z_{0}\right)\right\| \geqslant \bar{V}$, then $U(z)$ leaves $N_{1}$ in backward time.

Proof. Suppose that $\left\|V\left(z_{0}\right)\right\|>\bar{V}$ where $\bar{V}$ is to be determined. Then either

$$
\begin{aligned}
& V_{1}\left(z_{0}\right)>\frac{1}{2} \bar{V}, \quad V_{1}\left(z_{0}\right)<-\frac{1}{2} \bar{V}, \\
& V_{2}\left(z_{0}\right)>\frac{1}{2} \bar{V}, \quad \text { or } \quad V_{2}\left(z_{0}\right)<-\frac{1}{2} \bar{V} .
\end{aligned}
$$

We suppose that $V_{1}\left(z_{0}\right)>\frac{1}{2} \bar{V}$, and for convenience $z_{0}=0$. Choose $M_{1}$ so that $\|\nabla F(V)\|<M$ in $N_{1}$. Then, from (1A.5),

$$
V_{1}^{\prime}=\theta V_{1}-F_{u_{1}}(U) \leqslant T V_{1}+M_{1}
$$

as long as $V_{1} \geqslant 0$. Therefore, if $V_{1} \geqslant 0$,

$$
\left[e^{-T z} V_{1}\right]^{\prime} \leqslant e^{-T z} M_{1}
$$

Integrate this equation for $-z_{1} \leqslant z \leqslant 0$ to obtain

$$
V_{1}\left(-z_{1}\right) \geqslant e^{-T z_{1}} V_{1}(0)-\frac{1}{T} M_{1}\left(1-e^{-T z_{1}}\right) \geqslant \frac{1}{2} e^{-T} \bar{V}-\frac{1}{T} M_{1}
$$

as long as $0 \leqslant z_{1} \leqslant 1$ and $V_{1}(z) \geqslant 0$ for $-1 \leqslant z \leqslant 0$. This last statement is true if $\frac{1}{2} e^{-T} \bar{V}-(1 / T) M_{1}>0$ or $\bar{V}>(2 / T) M_{1} e^{+T}$, which we assume to be true. Therefore

$$
U_{1}^{\prime}(z)=V_{1}(z) \geqslant \frac{1}{2} e^{-T} \bar{V}-\frac{1}{T} M_{1}
$$

for $-1 \leqslant z \leqslant 0$. This implies that

$$
U_{1}(-1) \leqslant U_{1}(0)-\left(\frac{1}{2} e^{-T} \bar{V}-\frac{1}{T} M_{1}\right)
$$

Let $M_{2}=$ diameter of $N_{1}$ and choose $\bar{V}$ so that

$$
\frac{1}{2} e^{-T} \bar{V}-\frac{1}{T} M_{1}>M_{2} \text {. }
$$

Then (2D.2) and (2D.3) imply that $U_{1}(-1) \notin N_{1}$. Similar arguments hold for the other cases in (2D.1).

An immediate consequence of this result, Corollary 2A.2, and Lemma 2A.3 is

Corollary 2D.2. If $(U(z), V(z))$ is a solution of (1A.5), (1A.6), then $\theta<T$ and $(U(z), V(z)) \in N$ for all $z$.

E. The critical point $C$. From (2B.2) and (2B.3) it follows that $H(z)<F(B)<F(C)$ on all solutions of (1A.5), (1A.6). This implies that there exists $\delta$ such that if 
$(U(z), V(z))$ is a solution of (1A.5), (1A.6) then $U(z) \notin\{U:\|U-C\|<\delta\}=C_{\delta}$ for each $z$. Hence, the values of $F(U)$ in $C_{\delta}$ do not matter if we are only interested in solutions of (1A.5), (1A.6). In particular, $F(U)$ may be chosen to be arbitrarily large in $C_{\delta}$. We change $F(U)$ in $C_{\delta}$ so that if $(U(z), V(z)) \in W_{A^{\theta}}$ with $\theta<T$, then $U(z) \neq C$ for all $z$. This is possible for the following reason. Suppose that $(U(z), V(z)) \in W_{A^{\theta}}$ and $U\left(z_{0}\right)=C$ for some $z_{0}$. If $F(C)$ is very large, then we must have $\left\|V\left(z_{1}\right)\right\|$ very large for some $z_{1}<z_{0}$. However, as Proposition 2D.1 shows, if $\left\|V\left(z_{1}\right)\right\|$ is too large, then $U(z)$ will leave $N_{1}$ in backward time. The remark in $\S 2 \mathrm{~A}$ implies that $U(z)$ can never return to $N_{1}$, in backward time, after leaving $N_{1}$. This contradicts the assumption that $(U(z), V(z)) \in W_{A^{\theta}}$. It is very tedious to make this all precise so we do not give the details.

\section{The local unstable manifold at $(A, \mathcal{O})$ and the winding number.}

A. A parameterization of $W_{A^{\theta}}$. As before, let $W_{A^{\theta}}$ be the unstable manifold at $(A, \mathcal{O})$ for a particular $\theta$. As we saw in $\S 2 \mathrm{C}, \operatorname{dim} W_{A^{\theta}}=2$ for all $\theta$. The eigenvalues of the linearized equations at $(A, \mathcal{O})$ are given in $(2 \mathrm{C} .2)$, and their corresponding eigenvectors given in (2C.3). We conclude from the Stable Manifold Theorem (see [2]),

TheOREM 3A.1. Near $A^{\prime}=(A, \mathcal{O}), W_{A^{\theta}}$ is a $C^{2}$ injectively immersed, two-dimensional manifold. Moreover, the tangent space to $W_{A^{\theta}}$ at $A^{\prime}$ is the linear subspace spanned by $p_{1}^{+}$and $p_{2}^{+}$.

An important consequence of this theorem is

Proposition 3A.2. There exists $\delta>0$ such that if $A_{\delta}=\{U:\|A-U\|=\delta\}$ then for each $\theta$, we have that

(a) For each $U_{0} \in A_{\delta}$ there exists a unique $V_{0} \in \mathbf{R}^{2}$ such that $\left(U_{0}, V_{0}\right) \in W_{A^{\theta}}$.

(b) If $(U(z), V(z))$ is any nontrivial trajectory in $W_{A^{\theta}}$ then there exists a unique $z_{0}$ such that $U\left(z_{0}\right) \in A_{\delta}$.

This proposition implies that for each $\theta$ we may parametrize the nontrivial trajectories in $W_{A^{\theta}}$ by the points on $A_{\delta}$. Let us parametrize the points on $A_{\delta}$ by the angle $\varphi$. Let

$$
D_{1}=\{(\varphi, \theta): 0 \leqslant \varphi<2 \pi, 0<\theta \leqslant T\} .
$$

Then to each $(\varphi, \theta) \in D_{1}$ there corresponds a unique trajectory in $W_{A^{\theta}}$. If $(\theta, \varphi)=d$, then we denote this trajectory by $\gamma(d)(z)=(U(d)(z), V(d)(z))$. Here, $z$ is the independent variable along the trajectory.

B. The winding number. We wish to define the number of times trajectories in $W_{A^{\theta}}$ wind around $P_{D}$ and $P_{E}$. To do this let

$$
\begin{aligned}
& Q_{D}=\left\{(U, V): U_{1}=D_{1}, V_{1}<0, \text { and } U \in N\right\}, \\
& Q_{E}=\left\{(U, V): U_{1}=E_{1}, V_{1}>0, \text { and } U \in N\right\} .
\end{aligned}
$$

If $(U(z), V(z)) \in W_{A^{\theta}}$, let

$$
h(U, V)=\operatorname{card}\left\{z:(U(z), V(z)) \in Q_{D} \cup Q_{E}\right\} .
$$

By card $X$ we mean the cardinality of the set $X$. If $d \in \mathscr{D}_{1}$, let

$$
h(d)=h(\gamma(d)(z)) \text {. }
$$


REMARK. $h(d)$ counts the number of times $\gamma(d)(z)$ intersects $Q_{D} \cup Q_{E}$ which is equal to the number of times $\gamma(d)(z)$ winds around $P_{D}$ and $P_{E}$. This notion of winding number may seem complicated because it involves trajectories in fourdimensional space. However, one can compute $h(U, V)$ by considering $U(z)$ in the two-dimensional state space. Recall that $h(U, V)$ equals the number of times $(U, V)$ intersects $Q_{D}$ and $Q_{E}$. Now $\left(U\left(z_{0}\right), V\left(z_{0}\right)\right) \in Q_{D}$ if and only if $U\left(z_{0}\right) \in l_{D}$ and at $z=z_{0}, U(z)$ crosses $l_{D}$ from right to left. Similarly, $\left(U\left(z_{0}\right), V\left(z_{0}\right)\right) \in Q_{E}$ if and only if $U\left(z_{0}\right) \in l_{E}$ and at $z=z_{0}, U(z)$ crosses $l_{E}$ from left to right.

C. $\theta$ near 0 . Crucial to the proof of the theorem is the following result.

Proposition 3C.1. Given $M$ there exists $\theta_{M}$ such that if $0<\theta<\theta_{M}, 0 \leqslant \varphi<2 \pi$, $d=(\varphi, \theta)$, and $U(d)\left(z_{0}\right)=B$ for some $z_{0}$, then $h(d)>M$.

The proof of this result is quite technical so we save the proof for Appendix A.

\section{An algebraic invariant.}

A. A preliminary result.

Lemma 4A.1. Fix $\theta \in[0, T]$ and $q \in \partial X_{2}$, where $X_{2}$ was defined in (1B.1). Then there exists $\varphi=\varphi(\theta, q)$ such that $U(\varphi(\theta, q), \theta)\left(z_{0}\right)=q$ for some $z_{0}$ and $U(\varphi(\theta, q), \theta)(z) \in X_{2}$ for $z<z_{0}$. Moreover, $\varphi(\theta, q)$ can be chosen to depend continuously on $\theta$ and $q$.

The proof of this result is quite technical so we save the proof for Appendix B.

Let $q_{1}$ and $q$ be any points on $\partial X_{2}$ as shown in Figure 2. That is, $q_{1}$ is on the top side of $N_{1}$ and $q_{2}$ is on the bottom side. From Lemma 4A.1 there exists continuous functions $\varphi_{1}(\theta)$ and $\varphi_{2}(\theta)$ such that for $i=1,2, U\left(\varphi_{i}(\theta), \theta\right)(z)$ leaves $X_{2}$ through $q_{i}$. We assume, without loss of generality, that $\varphi_{1}(\theta)<\varphi_{2}(\theta)$ for all $\theta$. Let

$$
\begin{aligned}
& D=\left\{(\varphi, \theta) \in D_{1}: \varphi_{1}(\theta) \leqslant \varphi \leqslant \varphi_{2}(\theta)\right\}, \\
& X=\{d \in D: \gamma(d)(z) \rightarrow(B, \mathcal{O}) \text { as } z \rightarrow \infty\}, \\
& Y=D \backslash X .
\end{aligned}
$$

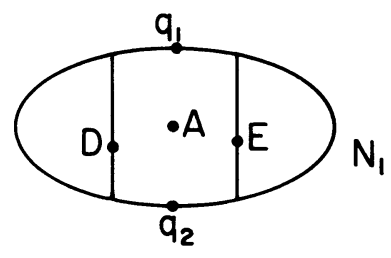

Figure 2

Note that $X$ corresponds to solutions of (1A.5) and (1A.6). We wish to prove that $X$ is an infinite set.

B. $\Gamma$ and $\Gamma^{*}$. Suppose that $y \in Y$, which was defined in the preceding section. We claim that $\gamma(y)(z)$ must leave $N$. From Propositin 2B.1 and the fact that $H(z)$ is increasing on solutions, it follows that if $\gamma(y)(z)$ does not leave $N$, then either $\lim _{z \rightarrow \infty} \gamma(y)(z)=(B, \mathcal{O})$ or $\lim _{z \rightarrow \infty} \gamma(d)(z)=(C, \mathcal{O})$. By the definition of $Y$, we 
have that $\lim _{z \rightarrow \infty} \gamma(y)(z) \neq(B, \mathcal{O})$. Moreover, the remarks in $\S 2 \mathrm{E}$ imply that $\lim _{z \rightarrow \infty} \gamma(y)(z) \neq(C, \mathcal{O})$. Hence, $\gamma(y)(z)$ leaves $N$. Let

$$
\mathscr{E}=\{(U, V) \in \partial N:\|V\|<\bar{V}\} \backslash\left(P_{D} \cup P_{E}\right) .
$$

From Proposition 2D.1 we conclude that if $y \in Y$, then $\gamma(y)(z)$ must leave $N$ through $\mathscr{E}$. Hence, we have a mapping $\Lambda: Y \rightarrow \mathscr{E}$ defined by $\Lambda(y)=$ the place where $\gamma(y)(z)$ leaves $N$. From Lemma 2A.3 it follows that $\Lambda$ is continuous.

Let $I$ be the unit interval and $\mathscr{G}$ the set of functions $g: I \rightarrow Y$ such that

(a) $g$ is continuous,

(b) $g(0) \in\left\{(\varphi, \theta): \varphi=\varphi_{1}(\theta)\right\}$,

(c) $g(1) \in\left\{(\varphi, \theta): \varphi=\varphi_{2}(\theta)\right\}$.

If $g \in \mathscr{G}$, then we have a continuous map $\Lambda \cdot g: I \rightarrow \mathscr{E}$. Note that $\mathscr{E}$ is topologically an annulus with four holes removed.

We now define two algebraic objects, $\Gamma^{*}(g)$ and $\Gamma(g)$, which indicate how the curve $(\Lambda \cdot g)(I)$ winds around the four holes. They will be elements of $F_{4}$, the set of words on the four elements $\alpha, \beta, \gamma$, and $\delta$.

We begin with some notation. For convenience we assume that $N_{1}$ is the square

$$
N_{1}=\left\{\left(U_{1}, V_{2}\right):\left|U_{1}\right| \leqslant W,\left|U_{2}\right| \leqslant W\right\} .
$$

Let

$$
\begin{gathered}
E_{1}=\left\{(U, V) \in \mathscr{E}: U_{1}>E_{1}\right\}, \\
E_{2}=\left\{(U, V) \in \mathscr{E}: D_{1}<U_{1}<E_{1}, U_{2}=W\right\}, \\
E_{3}=\left\{(U, V) \in \mathscr{E}: U_{1}<D_{1}\right\}, \\
E_{4}=\left\{(U, V) \in \mathscr{E}: D_{1}<U_{1}<E_{1}, U_{2}=-W\right\}, \\
l_{1}=l_{\alpha}^{+}=\left\{(U, V): U_{1}=E_{1}, U_{2}=W, 0<V_{1} \leqslant \bar{V}, V_{2}=0\right\}, \\
l_{2}=l_{\alpha}^{-}=\left\{(U, V): U_{1}=E_{1}, U_{2}=W,-\bar{V} \leqslant V_{1}<0, V_{2}=0\right\}, \\
l_{3}=l_{\beta}^{+}=\left\{(U, V): U_{1}=D_{1}, U_{2}=W, 0<V_{1} \leqslant \bar{V}, V_{2}=0\right\}, \\
l_{4}=l_{\beta}^{-}=\left\{(U, V): U_{1}=D_{1}, U_{2}=W,-\bar{V} \leqslant V_{1}<0, V_{2}=0\right\}, \\
l_{5}=l_{\gamma}^{+}=\left\{(U, V): U_{1}=D_{1}, U_{2}=-W, 0<V_{1} \leqslant \bar{V}, V_{2}=0\right\}, \\
l_{6}=l_{\gamma}^{-}=\left\{(U, V): U_{1}=D_{1}, U_{2}=-W,-\bar{V} \leqslant V_{1}<0, V_{2}=0\right\}, \\
l_{7}=l_{\delta}^{+}=\left\{(U, V): U_{1}=E_{1}, U_{2}=-W, 0<V_{1} \leqslant \bar{V}, V_{2}=0\right\}, \\
l_{8}=l_{\delta}^{-}=\left\{(U, V): U_{1}=E_{1}, U_{2}=-W,-\bar{V} \leqslant V_{1}<0, V_{2}=0\right\} .
\end{gathered}
$$

Assume that $g \in \mathscr{G}$. Choose $\eta_{k} \in[0,1], k=1,2, \ldots, K$, such that

(a) $\eta_{1}=0, \eta_{K}=1$,

(b) $\eta_{k}<\eta_{k+1}$ for all $k$,

(c) $(\Lambda \cdot g)\left(\eta_{k}\right) \in \bigcup_{i=1}^{4} E_{i}$ for all $k$,

(d) $(\Lambda \cdot g)\left(\eta_{k}, \eta_{k+1}\right)$ intersects at most one of the line segments $l_{i}, i=1, \ldots, 8$, for all $k$. We refer to $\eta^{*}=\left\{\eta_{1}, \ldots, \eta_{K}\right\}$ as a $g$-partition. It is not hard to prove that a $g$-partition does exist. 
548

DAVID TERMAN

TABLE 1

\begin{tabular}{|c|c|c|c|r|}
\hline$\Phi\left(\eta_{k}\right) \in$ & $\Phi\left(\eta_{k+1}\right) \in$ & $\begin{array}{c}\phi(\eta) \text { crosses } \\
\text { for } \eta \in\left(\eta_{k}, \eta_{k+1}\right)\end{array}$ & $\lambda\left(\eta_{k+1}\right)$ & $e\left(\eta_{k+1}\right)$ \\
\hline$E_{2}$ & $E_{1}$ & $l_{\alpha}^{+}$ & $\alpha$ & 1 \\
$E_{1}$ & $E_{2}$ & $l_{\alpha}^{+}$ & $\alpha$ & -1 \\
$E_{2}$ & $E_{3}$ & $l_{\beta}^{-}$ & $\beta$ & 1 \\
$E_{3}$ & $E_{2}$ & $l_{\beta}^{-}$ & $\beta$ & -1 \\
$E_{3}$ & $E_{4}$ & $l_{\gamma}^{-}$ & $\gamma$ & -1 \\
$E_{4}$ & $E_{3}$ & $l_{\gamma}^{-}$ & $\gamma$ & 1 \\
$E_{4}$ & $E_{5}$ & $l_{\delta}^{+}$ & $\delta$ & 1 \\
$E_{5}$ & $E_{4}$ & $l_{\delta}^{+}$ & $\delta$ & -1 \\
\hline
\end{tabular}

We now define $\Gamma^{*}\left(g, \eta^{*}\right)$. First we define $\lambda_{k}=\lambda\left(\eta_{k}\right) \in\{\alpha, \beta, \gamma, \delta\}$ and $e_{k}=$ $e\left(\eta_{k}\right) \in\{-1,0,1\}$. These quantities are determined by Table 1 . In the table, we let, for $s \in[0,1], \Phi(s) \equiv(\Lambda \cdot g)(s)$. For each case not shown in the chart we let $e_{k}=e\left(\eta_{k}\right)=0$. For this case we do not define $\lambda_{k}=\lambda\left(\eta_{k}\right)$ because, as we shall see, since $e_{k}=0$ the choice of $\lambda_{k}$ does not matter. Then define

$$
\Gamma^{*}\left(g, \eta^{*}\right)=\prod_{i=1}^{K} \lambda_{i}^{e_{i}} \stackrel{\text { def }}{=} \lambda_{1}^{e_{1}} \lambda_{2}^{e_{2}} \cdots \lambda_{K}^{e_{K}}
$$

An example is shown in Figure 3. In the figure, $\xi_{k}=\Phi\left(\eta_{k}\right)$. For this example

$$
\Gamma^{*}\left(g, \eta^{*}\right)=\alpha \alpha^{-1} \alpha \beta \gamma^{-1} \delta^{-1} \text {. }
$$
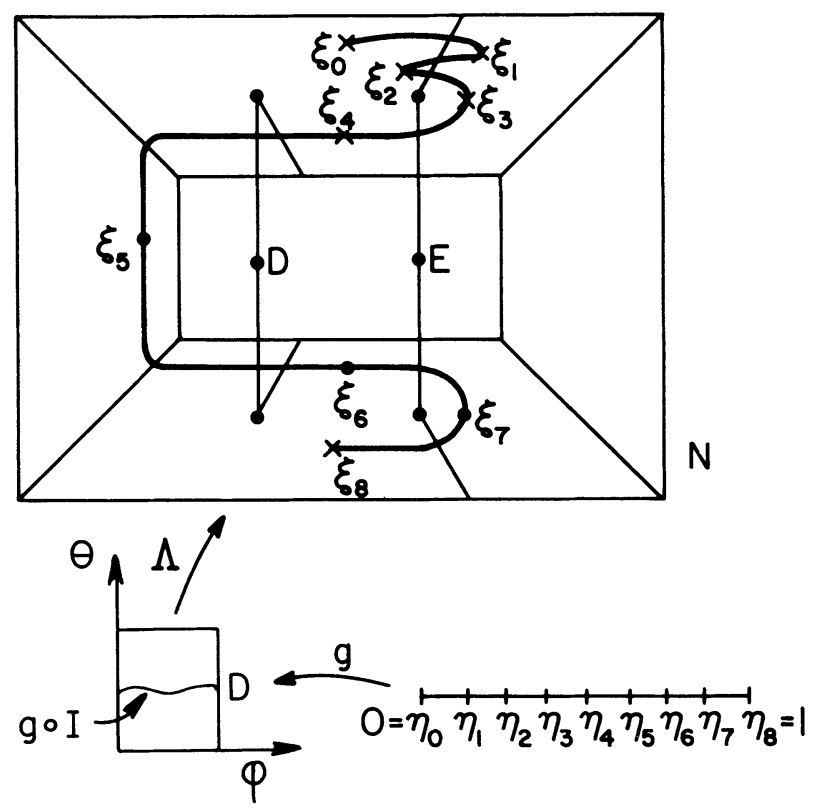

FIGURE 3 
Note that there may be cancellations in $\Gamma^{*}\left(g, \eta^{*}\right)$. By $\Gamma(g)$ we mean the element of $F_{4}$ obtained by making all cancellations in $\Gamma^{*}\left(g, \eta^{*}\right)$. We shall see later that $\Gamma(g)$ does not depend on $\eta^{*}$. In the above example $\Gamma(g)=\alpha \beta \gamma^{-1} \delta^{-1}$.

By $\Gamma^{*}(g)$ we mean the subset of $F_{4}$ consisting of all elements which yield, after all cancellations, $\Gamma(g)$. Note that, for each $\eta^{*}, \Gamma^{*}\left(g, \eta^{*}\right) \in \Gamma^{*}(g)$ and $\Gamma(g) \in \Gamma^{*}(g)$. For the above example, $\Gamma^{*}(g)$ includes the elements $\alpha \beta \delta \delta^{-1} \gamma^{-1} \delta^{-1}$ and $\alpha \beta \beta^{-1} \gamma \delta \delta^{-1} \gamma^{-1} \beta \gamma^{-1} \delta^{-1}$.

The following two propositions will be important for the rest of the paper. Their proofs are tedious but straightforward. We do not give the details.

Proposition 4B. $\Gamma(g)$ does not depend on the choice of $\eta^{*}$.

Before stating the next proposition we need the following definition.

Definition. Suppose that $g_{1}, g_{2} \in \mathscr{G}$. We say that $g_{1}$ is homotopic to $g_{2}$ relative to $Y$, and write $g_{1} \sim g_{2}$, if there exists a continuous map $\Phi: I \times I \rightarrow Y$ such that

(a) $\Phi(s, 0)=g_{1}(s)$ for $s \in I$,

(b) $\Phi(s, 1)=g_{2}(s)$ for $s \in I$,

(c) $\Phi(\cdot, t) \in \mathscr{G}$ for each $t \in I$.

Proposition 4B.2. If $g_{1} \sim g_{2}$, then $\Gamma\left(g_{1}\right)=\Gamma\left(g_{2}\right)$.

C. The winding number revisited. Recall that for each $d=(\varphi, \theta) \in D$ there corresponds a trajectory $\gamma(d)(z) \in W_{A^{\theta}}$. Moreover there is a winding number, $h(d)$, which counts the number of times $\gamma(d)(z)$ winds around $P_{D}$ and $P_{E}$. Suppose that $g(s) \in \mathscr{G}$ and $s_{0} \in I$. In this section we derive a formula for $h_{1}\left(s_{0}\right) \equiv h\left(g\left(s_{0}\right)\right)$ in terms of $\Gamma^{*}\left(g, \eta^{*}\right)$ for some $g$-partition $\eta^{*}$. First we need some notation.

Suppose that $\Gamma \in F_{4}$ is given by $\Gamma=\lambda_{1}^{e_{1}} \cdots \lambda_{f}^{e_{f}}$ where each $\lambda_{i} \in\{\alpha, \beta, \gamma, \delta\}$ and $e_{i} \in\{1,-1\}$. Let $\omega(\Gamma)=\sum_{i=1}^{J} e_{i}$. Let $g \in \mathscr{G}$ and $\eta^{*}$ be a $g$-partition. Define the map $\Lambda_{0}\left(g, \eta^{*}\right): I \rightarrow F_{4}$ as follows. Suppose that $\eta^{*}=\left\{\eta_{1}, \ldots, \eta_{K}\right\}$ and $\eta_{K} \leqslant s<$ $\eta_{k+1}$. Then define

$$
\Lambda_{0}\left(g, \eta^{*}\right)(s)=\prod_{i=1}^{k} \lambda_{i}^{e_{i}}=\lambda_{1}^{e_{1}} \cdots \lambda_{k}^{e_{k}} .
$$

The $\lambda_{i}$ and $e_{i}$ are defined as in the previous section. Finally, define $\Lambda_{1}\left(g, \eta^{*}\right): I \rightarrow$ $Z^{+}$, where $Z^{+}$is the set of nonnegative integers, by

$$
\Lambda_{1}\left(g, \eta^{*}\right)(s)=\left[\omega \cdot \Lambda_{0}\left(g, \eta^{*}\right)\right](s) .
$$

We can now state the main result of this section.

Proposition 4C.1. Assume that $g \in \mathscr{G}$ and $\eta^{*}=\left(\eta_{1}, \ldots, \eta_{K}\right)$ is a g-partition. If $\eta_{j} \leqslant s_{0}<\eta_{j+1}$, then either

$$
h_{1}\left(s_{0}\right)=\Lambda_{1}\left(g, \eta^{*}\right)\left(\eta_{j}\right) \quad \text { or } \quad h_{1}\left(s_{0}\right)=\Lambda_{1}\left(g, \eta^{*}\right)\left(\eta_{j+1}\right) .
$$

Proof. The proof is by induction on $j$. First assume that $j=1$. That is

$$
0=\eta_{1} \leqslant s_{0}<\eta_{2} \text {. }
$$

By assumption, $\eta_{1}=0$ and $(\Lambda \cdot g)\left(\eta_{1}\right) \in E_{2}$ where $E_{2}$ was defined in the preceding section. There are a number of cases to consider. Suppose, for example, that

$$
(\Lambda \cdot g)\left(s_{0}\right) \in E_{2} \quad \text { and } \quad(\Lambda \cdot g)\left(\eta_{2}\right) \in E_{2} .
$$


Because $(\Lambda \cdot g)\left(\eta_{2}\right) \in E_{2}$ it follows, from Table 1 , that $e_{1}=0$ and, therefore,

$$
\Lambda_{1}\left(g, \eta^{*}\right)\left(\eta_{1}\right)=\Lambda_{1}\left(g, \eta^{*}\right)\left(\eta_{2}\right)=0 \text {. }
$$

Hence, we need to prove that $h_{1}\left(s_{0}\right)=0$. Suppose that $h_{1}\left(s_{0}\right)>0$. Then $\gamma\left(g\left(s_{0}\right)(z)\right.$ must intersect $Q_{D} \cup Q_{E}$ at least once. Suppose that $\gamma\left(g\left(s_{0}\right)\right)(z)$ intersects $Q_{D}$. The other case is similar. Recall that

$$
Q_{D}=\left\{(U, V): U_{1}=D_{1}, V_{1}<0 \text {, and } U \in N_{1}\right\} \text {. }
$$

Because $(\Lambda \cdot g)\left(s_{0}\right) \in E_{2}$ it follows that $\gamma\left(g\left(s_{0}\right)\right)(z)$ must also intersect

$$
Q_{D}^{+}=\left\{(U, V): U_{1}=D_{1}, V_{1}>0 \text {, and } U \in N_{1}\right\} \text {. }
$$

Let

$$
\begin{aligned}
& s_{1}=\inf \left\{s: \gamma(g(s))(z) \text { intersects } Q_{D}\right\}, \\
& s_{2}=\inf \left\{s: \gamma(g(s))(z) \text { intersects } Q_{D}^{+}\right\} .
\end{aligned}
$$

Clearly, $0<s_{1}<s_{2}<s_{0}<\xi_{2}$. Moreover, $(\Lambda \cdot g)\left(s_{1}\right) \in l_{\beta}^{-}$and $(\Lambda \cdot g)\left(s_{2}\right) \in l_{\beta}^{+}$. This, however, contradicts the assumption that $\eta^{*}$ is a $g$-partition.

There are other cases to consider besides (4C.2). We only consider one more. The rest are similar. Suppose that $(\Lambda \cdot g)\left(s_{0}\right) \in E_{1}$ and $(\Lambda \cdot g)\left(\eta_{2}\right) \in E_{1}$. Then, using Table $1, \Lambda_{1}\left(g, \eta^{*}\right)\left(\eta_{1}\right)=0$ and $\Lambda_{1}\left(g, \eta^{*}\right)\left(\eta_{2}\right)=1$. We claim that $h_{1}\left(s_{0}\right)=1$. Because $(\Lambda \cdot g)\left(\eta_{1}\right) \in E_{2}$ and $(\Lambda \cdot g)\left(s_{0}\right) \in E_{1}$ it is clear that $h_{1}\left(s_{0}\right) \neq 0$. Suppose that $h_{1}\left(s_{0}\right)>1$. Then $\gamma\left(g\left(s_{0}\right)\right)(z)$ must intersect $Q_{D} \cup Q_{E}$ at least twice. Because $\gamma(g(0))(z)$ does not intersect $Q_{D} \cup Q_{E}$ at all, this implies that there exist $s_{1}, s_{2}$ with $\eta_{1}<s_{1}<s_{2}<s_{0}<\eta_{2}$ such that $(\Lambda \cdot g)\left(s_{1}\right) \in l_{i}$ and $(\Lambda \cdot g)\left(s_{2}\right) \in l_{j}$ for $i \neq j$. This, however, contradicts the assumption that $\eta^{*}$ is a $g$-partition.

To complete the proof of the proposition we must prove the induction step. That is, we assume that the proposition is true if $\eta_{j} \leqslant s_{0} \leqslant \eta_{j+1}$ for $j<k$, and then prove the result if $j=k$. The proof of this is very similar to the proof just given so we do not include the details.

\section{Completion of the proof of Theorem 1.}

A. Preliminaries. Suppose that $g \in \mathscr{G}$, which was defined in the previous section, and

$$
\Gamma(g)=\lambda_{1}^{e_{1}} \lambda_{2}^{e_{2}} \cdots \lambda_{K}^{e_{K}}
$$

where, for each $i, \lambda_{i} \in\{\alpha, \beta, \gamma, \delta\}$ and $e_{i} \in\{-1,1\}$. Define

$$
|\Gamma(g)|=\sup _{1 \leqslant J \leqslant K} \sum_{i=1}^{J} e_{i} .
$$

In the next subsection we prove

Proposition 5A.1. Let $M$ be any positive integer and let $\theta_{M}$ be as in Proposition 3C.1. Suppose that $g \in \mathscr{G}$ is given by $g(s)=(\varphi(s), \theta(s))$ and $\theta(s)<\theta_{M}$ for each $s$. Then $|\Gamma(g)|>M$.

In this section we show that Proposition 5A.1 implies that Theorem 1 is true.

Suppose that Theorem 1 is not true; that is, there exists only a finite number of $\theta$ 's, say $\left\{\theta_{1}, \ldots, \theta_{N}\right\}$, for which there exists a solution of (1A.5), (1A.6). Let

$$
\boldsymbol{\theta}_{0}=\frac{1}{2} \inf _{1 \leqslant j \leqslant N} \boldsymbol{\theta}_{j} .
$$


Then $\theta_{0}>0$. Choose $g_{0} \in \mathscr{G}$ such that $g_{0}(s)=(\varphi(s), \theta(s))$ and $\theta(s)=\theta_{0}$ for all $s$. From Proposition 5A.1 there exists $\theta^{*}<\theta_{0}$ such that if $g_{1}(s) \in \mathscr{G}$ is such that $g_{1}(s)=\left(\varphi_{1}(s), \theta_{1}(s)\right)$ and $\theta_{1}(s)=\theta^{*}$ for all $s$, then $\left|\Gamma\left(g_{1}\right)\right|>\left|\Gamma\left(g_{0}\right)\right|+1$. But $g_{0}(s)$ and $g_{1}(s)$ are clearly homotopic relative to $Y$. From Proposition 4G.2 it follows that $\Gamma\left(g_{0}\right)=\Gamma\left(g_{1}\right)$. Hence,

$$
\left|\Gamma\left(g_{0}\right)\right|=\left|\Gamma\left(g_{1}\right)\right|>\left|\Gamma\left(g_{0}\right)\right|+1 \text {. }
$$

This is clearly impossible, thus proving the theorem.

B. Proof of Proposition 5A.1. Let $\gamma(g(s))(z)=(U(g(s))(z), V(g(s))(z))$. Now $U(g(0))(z)$ leaves $X_{2}$ through its top side without ever crossing $l_{D}$ or $l_{E}$, and $U(g(1))(z)$ leaves $X_{2}$ through its bottom side without ever crossing $l_{D}$ or $l_{E}$. Moreover, by the remarks in $\S 2 \mathrm{E}, U(g(s))(z) \neq C$ for all $s$ and $z$. Since the curves $U(g(s))(\cdot)$ vary continuously with $s$ this implies that there exist $s_{0}$ and $z_{0}$ such that $U\left(g\left(s_{0}\right)\right)\left(z_{0}\right)=B$. Because $\theta\left(s_{0}\right)<\theta_{M}$, Proposition 3C.1 implies that $h_{1}\left(s_{0}\right)=$ $h\left(g\left(s_{0}\right)\right)>M$. By Proposition 4C.1, if $\eta^{*}$ is a $g_{0}$-partition and $\eta_{j} \leqslant s_{0}<\eta_{j}$, then either

$$
h_{1}\left(s_{0}\right)=\Lambda_{1}\left(g, \eta^{*}\right)\left(\eta_{j}\right) \text { or } h_{1}\left(s_{0}\right)=\Lambda_{1}\left(g, \eta^{*}\right)\left(\eta_{j+1}\right) .
$$

From the definitions this implies that $\left|\Gamma^{*}\left(g, \eta^{*}\right)\right|>M$. We must show that this implies that $|\Gamma(g)|>M$.

Note that $\Gamma(g)$ is obtained from $\Gamma^{*}\left(g, \eta^{*}\right)$ by a finite number of cancellations. We show that after each cancellation the index is still greater than $M$. More precisely, suppose that $\Gamma^{*}\left(g, \eta^{*}\right)$ is of the form

$$
\Gamma^{*}\left(g, \eta^{*}\right)=\Gamma_{A}^{*} \lambda_{k}^{e_{k}} \lambda_{k+1}^{e_{k+1}} \Gamma_{B}^{*}
$$

where $\Gamma_{A}^{*}, \Gamma_{B}^{*} \in F_{4}, \lambda_{k}=\lambda_{k+1} \in\{\alpha, \beta, \gamma, \delta\}$, and $e_{k}=-e_{k+1} \in\{-1,1\}$. Let $\Gamma^{\prime}=$ $\Gamma_{A}^{*} \Gamma_{B}^{*}$. We prove that $\left|\Gamma^{\prime}\right|>M$. Since $\Gamma(g)$ is obtained from $\Gamma^{*}\left(g, \eta^{*}\right)$ by a finite number of such cancellations, this will prove the result.

Note that if $e_{k}=-1$, then $\left|\Gamma^{\prime}\right|=\left|\Gamma^{*}\left(g, \eta^{*}\right)\right|>M$. Hence, we may assume that $e_{k}=+1$.

There are four cases to consider. Either $\lambda_{k}=\alpha, \beta, \gamma$, or $\delta$. First suppose that $\lambda_{k}=\alpha$. We then consider two subcases. These are

(a) $h\left(g\left(\eta_{0}\right)\right)>M$ for some $\eta_{0}<\eta_{k-1}$.

(b) $h(g(\eta)) \leqslant M$ for all $\eta<\eta_{k-1}$.

Suppose (5B.2a). Choose $j<k-1$ such that $\eta_{j} \leqslant \eta_{0}<\eta_{j+1}$. Then, from Proposition 4C.1, either

$$
h\left(g\left(\eta_{0}\right)\right)=\sum_{i=1}^{j} e_{i}>M, \text { or } h\left(g\left(\eta_{0}\right)\right)=\sum_{i=1}^{j+1} e_{i}>M .
$$

In either case, it follows that $\left|\Gamma^{\prime}\right|>M$. This is what we wished to prove.

Now suppose that (5B.2b) holds. We first show that (5B.2b) implies that $U(g(\eta))(z) \neq B$ for all $z$ and $\eta<\eta_{k+1}$. Suppose, for the sake of contradiction, that $U\left(g\left(\eta_{0}\right)\right)\left(z_{0}\right)=B$ for some $z_{0}$ and $\eta_{0}<\eta_{k+1}$. Let

$$
t\left(\eta_{0}\right)=\sup \left\{z<z_{0}: U\left(g\left(\eta_{0}\right)\right)(z) \in l_{D}\right\} \text {. }
$$


Because $\lim _{z \rightarrow-\infty} U\left(g\left(\eta_{0}\right)(z)=A\right.$ and $U\left(g\left(\eta_{0}\right)\right)\left(z_{0}\right)=B$, it is clear that $t\left(\eta_{0}\right)$ is well defined. For $\eta$ close to $\eta_{0}$ there exists a continuous function $t(\eta)$ such that $U(g(\eta))(t(\eta)) \in l_{D}$. Let $J$ be the maximum subset of $[0,1]$ such that $t(\eta)$ is a well defined, continuous function. Let $\zeta=\inf \{\eta: \eta \in J\}$. Because $U\left(g\left(\eta_{0}\right)\right)\left(z_{0}\right)=B$ we conclude from Proposition 3C.1 that $h\left(g\left(\eta_{0}\right)\right)>M$. It follows that $h(g(\eta))>M$ for all $\eta \in J$. In particular, $h(g(\zeta))>M$. If $\zeta<\eta_{k-1}$, then we have a contradiction of (5B.2b). Therefore, assume that $\eta_{k-1} \leqslant \zeta<\eta_{k+1}$. Clearly, $U(g(\zeta))(z)$ leaves $N_{1}$ through $l_{D} \cap \partial N_{1}$. Hence, $(\Lambda \cdot g)(\zeta) \in l_{\beta}^{-} \cup l_{\gamma}^{-}=l_{4} \cup l_{6}$, where $\Lambda, l_{\beta}^{-}, l_{\gamma}^{-}, l_{4}$ and $l_{6}$ were defined in $\S 4 \mathrm{~B}$. However, from the definition of a $g$-partition, $(\Lambda \cdot g)(\eta)$ can cross at most one of the lines $l_{1}-l_{8}$ for $\eta \in\left(\eta_{k}, \eta_{k+1}\right)$. Because $\lambda_{k}=\alpha$ and $\lambda_{k+1}=\alpha$ we have, from Table 1 , that $(\Lambda \cdot g)(\eta)$ crosses $l_{\alpha}^{+}=l_{1}$ for some $\eta \in$ $\left[\eta_{k-1}, \eta_{k}\right)$ and another $\eta \in\left[\eta_{k}, \eta_{k+1}\right)$. This gives the desired contradiction. We have now shown that if (5B.2b) holds, then $U(g(\eta))(z) \neq B$ for all $z$ and $\eta<\eta_{k+1}$.

It is clear that $U\left(g\left(\eta_{0}\right)\right)\left(z_{0}\right)=B$ for some $\eta_{0}$ and some $z_{0}$. If (5B.2b) holds then $\eta_{0}>\eta_{k+1}$. Suppose that $\eta_{j} \leqslant \eta_{0}<\eta_{j+1}$ for some $j \geqslant k+1$. Because $U\left(g\left(\eta_{0}\right)\right)=B$ it follows that $h\left(g\left(\eta_{0}\right)\right)>M$. From Proposition 4C.1 we conclude that either $\Lambda_{1}\left(g, \eta^{*}\right)\left(\eta_{j}\right)>M$ or $\Lambda_{1}\left(g, \eta^{*}\right)\left(\eta_{j+1}\right)>M$. Both of these inequalities imply that $\left|\Gamma^{\prime}\right|>M$.

It remains to consider the cases $\lambda_{k}=\beta, \gamma$, and $\delta$. The proofs in each of these cases is similar to the one just given so we do not include the details.

\section{Appendix A. Proof of Proposition 3C.1.}

Idea of the proof. Suppose that $(U(z), V(z)) \in W_{A^{\theta}}$ and $U\left(z_{0}\right)=B$ for some $z_{0}$. Then while $(U(z), V(z)) \in N, H(z)$ must increase from $F(A)$ to $F(B)$. Recall that $H^{\prime}(z)=\theta\|V\|^{2} \leqslant \theta \bar{V}^{2}$ as long as $(U, V) \in N$. Then $H(z)$ increases very slowly if $\theta$ is very small. Since $H(z)$ must increase from $F(A)$ to $F(B)$ this implies that $(U(z), V(z))$ must spend a long time in $N$. We shall use hypothesis (F6) to conclude that $U(z)$ must move back and forth between the mountain peaks defined by $F(A)$, $F(B)$, and $F(C)$ a large number of times. Together with the remarks in $\S 3 \mathrm{~B}$ this implies the desired result.

Proof of Proposition 3C.1. Let $\gamma(d)(z)=(U(z), V(z))$. We shall prove that there exists $\theta_{0}$ such that if $\theta<\theta_{0}$ and $U\left(z_{1}\right) \in X_{i}, i=1,2$, or 3 , then there exists $z_{2}>z_{1}$ such that $U\left(z_{2}\right) \notin X_{i}$ and $H\left(z_{2}\right)-H\left(z_{1}\right) \leqslant F(B) / 4 M$. This proves the proposition because it implies that $U(z)$ must change regions $\left(X_{1}, X_{2}\right.$, or $\left.X_{3}\right)$ at least $4 M$ times if $U\left(z_{0}\right)=F(B)$ for some $z_{0}$. Together with the remark in $\S 3 \mathrm{~B}$ this implies the desired result.

We begin with a rather technical result. For $r>0$ let $A_{r}=\{U:\|U-A\| \leqslant r\}$.

LEMMA A.1. There exists $\theta_{1}, h_{0}, r$ such that if $\theta<\theta_{1},\left(U\left(z_{1}\right), V\left(z_{1}\right)\right) \in \partial A_{r}$, $\left\langle U\left(z_{1}\right), V\left(z_{1}\right)\right\rangle>0$, and $H\left(z_{1}\right)<h_{0}$, then there exists $z_{2}>z_{1}$ such that $U\left(z_{2}\right) \notin X_{2}$ and $U(z) \notin A_{r}$ for $z \in\left(z_{1}, z_{2}\right)$.

Proof of Lemma A.1. Suppose that the lemma is not true. Then for each $n>0$ there exists $\theta_{n}, h_{n}, r_{n}$ which all approach zero as $n \rightarrow \infty$, solutions $\left(U_{n}(z), V_{n}(z)\right)$ of (1A.5) with $\theta=\theta_{n}$ and $z_{n}$ such that $\left(U_{n}\left(z_{n}\right), V_{n}\left(z_{n}\right)\right) \in \partial A r_{n},\left\langle U_{n}\left(z_{n}\right), V_{n}\left(z_{n}\right)\right\rangle>0$, 
$H\left(z_{n}\right)=h_{n}$, and each $U_{n}(z)$ returns to $A r_{n}$ before leaving $X_{2}$. By compactness, this implies that some subsequence of $\left\{U_{n}(z), V_{n}(z)\right\}$ converges to a solution $(U(z), V(z))$ of (1A.5) with $\theta=0$ such that $H(z)=0$ along $(U(z), V(z))$ and $\lim _{z \rightarrow \pm \infty} U(z)=A$. Hence, $(U(z), V(z))$ is a bounded solution of (1A.5) such that $U(z)$ lies entirely in $X_{2}$. If we show that $U(z) \neq A$ for some $z$, then this will contradict (F6).

Let $\delta$ be as in Proposition 3A.2. The assumption that $\left\langle U_{n}(z), V_{n}(z)\right\rangle>0$ for each $n$ and the saddle point property at $(A, \mathcal{O})$ imply that for each $n$ there exists $z_{n}^{\prime}$ such that $\left\|U_{n}\left(z_{n}^{\prime}\right)-A\right\|=\delta$. Hence, there exists $z^{\prime}$ such that $\left\|U\left(z^{\prime}\right)-A\right\|=\delta$. In particular, $U\left(z^{\prime}\right) \neq A$ and the proof of the lemma is complete. In what follows we assume that $h_{0}<F(B)$.

We now return to the proof of Proposition 3C.1.

First suppose that $i=1$ or 3 . Assumption (F6) implies that there exists $T_{1}$ such that if $\theta=0,0<H\left(z_{1}\right)<F(B) / 2$ and $U\left(z_{1}\right) \in X_{i}$, then $U(z) \notin X_{i}$ for some $z \in\left(z_{1}, z_{1}+T_{1}\right)$. By continuous dependence of solutions on a parameter it follows that there exists $\theta_{0}$ such that if $\theta<\theta_{0}, 0<H\left(z_{1}\right)<F(B) / 2$ and $U\left(z_{1}\right) \in X_{i}$, then $U(z) \notin X_{i}$ for some $z \in\left(z_{1}, z_{1}+2 T_{1}\right)$. Let

$$
z_{2}=\inf \left\{z>z_{1}: U(z) \notin X_{i}\right\} .
$$

Then

$$
\begin{aligned}
H\left(z_{2}\right)-H\left(z_{1}\right) & =\int_{z_{1}}^{z_{2}} H^{\prime}(z) d z=\int_{z_{1}}^{z_{2}} \theta\langle V(z), V(z)\rangle^{2} d z \\
& \leqslant \theta_{0} \bar{V}^{2} 2 T_{1} \leqslant h_{0} / 12 M<F(B) / 4 M
\end{aligned}
$$

if

$$
\theta_{0} \leqslant \frac{h_{0}}{24 M} \bar{V}^{2} T_{1}
$$

which we assume to be true.

It remains to consider the case when $U\left(z_{1}\right) \in X_{2}$ for some $z_{1}$. Note that $U(z)$ starts out in $X_{2}$ (because $\lim _{z \rightarrow-\infty} U(z)=A$ ). If $\theta=0$, then (F6) implies that there exists $\xi_{0}$ such that $U\left(\xi_{0}\right) \notin X_{2}$. Moreover, $H\left(\xi_{0}\right)=0$. Hence, $\theta_{0}$ can be chosen such that if $0<\theta<\theta_{0}$, then there exists $\xi_{1}$ such that $U\left(\xi_{1}\right) \notin X_{2}$ and $H\left(\xi_{1}\right)<h_{0} / 12 M$.

Let

$$
\eta_{1}=\inf \left\{z: U(z) \notin A_{r}\right\} \quad \text { and } \quad \eta_{2}=\inf \left\{z: U(z) \notin X_{2}\right\}
$$

Let

$$
\gamma=\min \left\{\left\|\gamma_{1}-\gamma_{2}\right\|: \gamma_{1} \in A, \gamma_{2} \notin X\right\} .
$$

Since $\|V(z)\| \leqslant \bar{V}$ it follows that $\eta_{2}-\eta_{1} \geqslant \gamma / \bar{V}$. Let

$$
-\lambda=\sup \{F(U(z)): U(z) \in \partial A r\}<0
$$

because we are assuming that $F(A)=0$. Then, for $z \in\left(\eta_{1}, \eta_{2}\right)$,

$$
0<H(z)=\frac{1}{2} F(U)+\|V(z)\|^{2} \leqslant-\frac{1}{2} \lambda+\|V(z)\|^{2},
$$

or

$$
\|V(z)\|^{2}>+\frac{1}{2} \lambda
$$


It follows that

$$
\begin{aligned}
H\left(\eta_{2}\right) & \geqslant H\left(\eta_{2}\right)-H\left(\eta_{1}\right)=\int_{\eta_{1}}^{\eta_{2}} H^{\prime}(\eta) d \eta \\
& \geqslant \int_{\eta_{1}}^{\eta_{2}} \theta\langle V(\eta), V(\eta)\rangle d \eta \\
& \geqslant \int_{\eta_{1}}^{\eta_{2}} \frac{\theta \lambda}{2} d \eta \geqslant \frac{\theta \lambda \gamma}{2 \bar{V}} .
\end{aligned}
$$

Therefore,

$$
H(z) \geqslant \frac{1}{2} \frac{\theta \lambda \gamma}{\bar{V}} \text { if } z>\eta_{2}
$$

Now suppose that $z_{1}>\eta_{2}$ and $U\left(z_{1}\right) \in X_{2}$. There are two cases to consider. These are, (1) $U\left(z_{2}\right) \notin X_{2}$ for some $z_{2}>z_{1}$ and $U(z) \notin A_{r}$ for $z \in\left(z_{1}, z_{2}\right)$, and (2) $U\left(z_{2}\right) \in A_{r}$ for some $z_{2}>z_{1}$ and $U(z) \in X_{2}$ for $z \in\left(z_{1}, z_{2}\right)$.

As before, (F6) and the continuous dependence of solutions on a parameter imply that $\theta_{0}$ and $T_{1}$ can be chosen so that if $\theta<\theta_{0}, z_{1}>\eta_{2}, U\left(z_{1}\right) \in X_{2}$, and $U(z) \notin A_{r}$ for $z \in\left(z_{1}, z_{1}+T_{1}\right)$, then there exists $z_{2}$ such that $z_{1}<z_{2}<z_{1}+T_{1}$ and $U\left(z_{2}\right) \notin$ $X_{2}$. As before, $H\left(z_{2}\right)-H\left(z_{1}\right)<h_{0} / 12 M$ if (A.1) is satisfied.

It remains to consider the case $U\left(z_{1}\right) \in X_{2}, z_{1}>\eta_{2}$, and there exists $z_{2}>z_{1}$ such that $U\left(z_{2}\right) \in A_{r}$ and $U(z) \in X_{2}$ for $z \in\left(z_{1}, z_{2}\right)$. We assume that

$$
z_{2}=\inf \left\{z>z_{1}: U(z) \in A_{r}\right\} \text {. }
$$

As before, $\theta_{0}$ and $T_{1}$ can be chosen so that if $\theta<\theta_{0}$, then $z_{2}-z_{1}<T_{1}$. Moreover, $H\left(z_{2}\right)-H\left(z_{1}\right) \leqslant h_{0} / 12 M$ if $\theta_{0}$ is sufficiently small. Because $z_{2}>\eta_{2}$, we have, from (A.2), that $H\left(z_{2}\right) \geqslant \lambda \theta \gamma / 2 \bar{V}$. Hence, as long as $U(z) \in A_{r},\|V(z)\|^{2} \geqslant$ $F(U) / 2+\|V\|^{2} \geqslant H(z) \geqslant \theta \lambda \gamma / 2 \bar{V} \equiv \theta K$. This implies that $U(z)$ must pass through $A r$. If we let $z_{3}=\inf \left\{z>z_{2}: U(z) \notin A_{r}\right\}$, then

$$
z_{3}-z_{2} \leqslant \frac{\text { diameter of } A r}{\inf \|V(z)\|} \leqslant \frac{2 r}{\theta^{1 / 2} K^{1 / 2}} .
$$

Moreover,

$$
\begin{aligned}
H\left(z_{3}\right)-H\left(z_{2}\right) & =\int_{z_{2}}^{z_{3}} H^{\prime}(z) d z \leqslant \theta \bar{V}^{2}\left(z_{3}-z_{2}\right) \\
& \leqslant \theta \bar{V}^{2} \frac{2 r}{\theta^{1 / 2} K^{1 / 2}} \leqslant \theta_{0}^{1 / 2} \frac{2 \bar{V}^{2} r}{K^{1 / 2}} \leqslant \frac{h_{0}}{12 M}
\end{aligned}
$$

if $\theta_{0}$ is sufficiently small.

From Lemma A.1 there exists $z_{4}>z_{3}$ such that $U\left(z_{4}\right) \notin X_{2}$ and $U(z) \notin A_{r}$ for $z \in\left(z_{3}, z_{4}\right)$. We assume that $z_{4}=\inf \left\{z>z_{3}: U(z) \notin X_{2}\right\}$. As before, $\theta_{0}$ and $T_{1}$ can be chosen so that if $\theta<\theta_{0}$, then $z_{4}-z_{3}<T_{1}$. Hence, if (A1.1) holds, then

$$
H\left(z_{4}\right)-H\left(z_{3}\right) \leqslant h_{0} / 12 M \text {. }
$$

We can now estimate the change of $H(z)$ as $U(z)$ crosses $X_{2}$ in the case that $U(z)$ crosses $A_{r}$. Let $z_{1}, z_{2}, z_{3}$ and $z_{4}$ be as above. Then

$$
\begin{aligned}
H\left(z_{4}\right)-H\left(z_{1}\right) & =\left[H\left(z_{4}\right)-H\left(z_{3}\right)\right]+\left[H\left(z_{3}\right)-H\left(z_{2}\right)\right]+\left[H\left(z_{2}\right)-H\left(z_{1}\right)\right] \\
& \leqslant h_{0} / 4 M \leqslant F(B) / 4 M,
\end{aligned}
$$

which is what we wished to prove. 
Appendix B. Proof of Lemma 4A.1. Let $I$ be equal to the set of $\theta \in[0, T]$ for which the first part of the lemma is true. That is, if $\theta \in I$ and $q \in \partial X_{2}$, then there exists $\varphi(\theta, q)$ such that $U(\varphi(\theta, q), \theta)\left(z_{0}\right)=q$ for some $z_{0}$ and $U(\varphi(\theta, q), \theta)(z) \in X_{2}$ for $z<z_{0}$. We prove that $I$ is nonempty, open, and closed.

We first prove that $I$ is open. Assume that $\theta_{0} \in I$. Note that for each $(\varphi, \theta) \in D_{1}$, if $\gamma(\varphi, \theta)(z)=(U(z), V(z))$, then $U(z)$ must leave $X_{2}$. This is because of assumption (F6). Moreover, if we set $Q(\varphi, \theta)$ equal to the place where $U(z)$ leaves $X_{2}$. then $Q(\varphi, \theta)$ is continuous. This is because $U(z)$ cannot be tangent to $\partial X_{2}$.

Fix $q_{0} \in \partial X_{2}$, and let $q_{1}$ and $q_{2}$ be any other distinct points on $\partial X_{2}$. Choose $\varphi_{1}$ and $\varphi_{2}$ such that $Q\left(\varphi_{1}, \theta_{0}\right)=q_{1}$ and $Q\left(\varphi_{2}, \theta_{0}\right)=q_{2}$. Then $\varphi_{1}$ and $\varphi_{2}$ split $\Sigma=$ $\left\{(\varphi, \theta): \theta=\theta_{0}\right\}$ into two distinct subsets, if we think of $\Sigma$ as a circle identifying $\varphi=0$ with $\varphi=2 \pi$. We label these two subsets by $\Sigma_{1}$ and $\Sigma_{2}$. Moreover, $q_{1}$ and $q_{2}$ split $\partial X_{2}$ into two distinct subsets which we label as $Y_{1}$ and $Y_{2}$. We assume that $q_{0} \in Y_{1}$. Then either $Q\left(\Sigma_{1}, \theta_{0}\right)$ covers $Y_{1}$ and $Q\left(\Sigma_{2}, \theta_{0}\right)$ covers $Y_{2}$, or $Q\left(\Sigma_{1}, \theta_{0}\right)$ covers $Y_{2}$ and $Q\left(\Sigma_{2}, \theta_{0}\right)$ covers $Y_{1}$. This follows from the assumption that $\theta_{0} \in I$. Suppose that $Q\left(\Sigma_{1}, \theta_{0}\right)$ covers $Y_{1}$. Let $q_{3}$ and $q_{4}$ be any other points in $Y_{1}$ such that the portion of $\partial X_{2}$ which lies in $Y_{1}$ and between $q_{3}$ and $q_{4}$ contains $q_{0}$. Call this subset of $\partial X_{2}, Y_{3}$. Since $Q(\varphi, \theta)$ is continuous, there exists $\lambda$ such that if $\left|\theta_{0}-\theta\right|<$ $\lambda$, then $Q\left(\Sigma_{1}, \theta\right)$ covers $Y_{3}$. Since $q_{0} \in Y_{3}$, if $\left|\theta-\theta_{0}\right|<\lambda$ then $Q(\varphi, \theta)=q_{0}$ for some $\varphi$. Since $\partial X_{2}$ is compact this implies that $\lambda$ can be chosen so that if $\left|\theta-\theta_{0}\right|<\lambda$, then $Q(\Sigma, \theta)$ covers $\partial X_{2}$. Hence, $I$ is open.

We now prove that $I$ is closed. Fix $q_{0} \in \partial X_{2}$ and $\theta_{0} \in[0, T]$. Assume there exists a sequence $\left\{\theta_{k}\right\}$ such that for each $k, \theta_{k} \in I$ and $\theta_{k} \rightarrow \theta_{0}$ as $k \rightarrow \infty$. Choose $\left\{\varphi_{k}\right\}$ so that $Q\left(\varphi_{k}, \theta_{k}\right)=q_{0}$. By compactness, some subsequence of $\left\{\varphi_{k}\right\}$ converges, to say $\varphi_{0}$. Clearly, $Q\left(\varphi_{0}, \theta_{0}\right)=q_{0}$. Since $q_{0}$ was arbitrary, $\theta_{0} \in I$ and $I$ is closed.

It remains to prove that $I$ is nonempty. We prove that $\theta \in I$ if $\theta$ is sufficiently large. We will prove that there exists $T_{1}$ such that if $\theta>T_{1}, \varphi \in[0,2 \pi), d=(\varphi, \theta)$ and $\gamma(d)(z)=(U(z), V(z))$, then $\|U(z)-A\|$ is increasing as long as $U(z) \in X_{2}$. In particular, $U(z) \neq A$ for each $z$. This certainly implies the desired result.

We wish to prove that there exists $T_{1}$ such that if $\theta>T_{1}$ and $(U(z), V(z)) \in W_{A^{\theta}}$, then $\|U(z)-A\|$ is increasing as long as $U(z) \in X_{2}$. Certainly there exists $\delta$ such that if $\|U(z)-A\|<\delta$ for $z<z_{0}$, and $\theta>0$, then $\|U(z)-A\|$ is increasing for $z<z_{0}$. Let

$$
\begin{aligned}
& M_{1}=\sup \{\|\nabla F(U)\|: U \in X\}, \\
& M_{2}=\sup \left\{|\langle U-A, \nabla F(U)\rangle|: U \in X_{2}\right\}, \\
& \lambda_{1}=\max \left\{2 M_{1}, 2 M_{2}^{1 / 2}\right\} .
\end{aligned}
$$

By Lemma 2C.2 there exists $T_{1}$ such that if $\theta>T_{1},\left\|U\left(z_{0}\right)-A\right\|=\delta$ and $\| U(z)-$ $A \|<\delta$ for $z<z_{0}$, then $\left\|V\left(z_{0}\right)\right\|>\lambda$. We assume that $T_{1}>1$. Then for each $z>z_{0}$, $\theta>T_{1}$,

$$
\begin{aligned}
\frac{d}{d z}\langle V, V\rangle & =2 \theta\langle V, V\rangle-2\langle V, \nabla F(U)\rangle \geqslant 2 \theta\|V\|^{2}-2\|V\| M_{1} \\
& \geqslant 2\|V\|\left(\theta\|V\|-M_{1}\right) \geqslant 2\|V\|\left(\lambda_{1}-M_{1}\right)>0
\end{aligned}
$$


as long as $\|V\|>\lambda_{1}$ and $U(z) \in X_{2}$. However, at $z=z_{0},\|V\|>\lambda_{1}$ and (5B.1) implies that $\|V\|$ is then increasing. Hence, $\|V(z)\| \geqslant \lambda$ for $z \geqslant z_{0}, U(z) \in X_{2}$. Another computation shows that for $z \geqslant z_{0}, \theta>T_{1}, U(z) \in X_{2}$,

$$
\begin{aligned}
\langle U-A, V\rangle^{\prime} & =\langle V, V\rangle+\theta\langle U-A, V\rangle-\langle U-A, \nabla F(U)\rangle \\
& >\lambda_{1}^{2}+T_{1}\langle U-A, V\rangle-M_{2}>3 M_{2}+T_{1}\langle U-A, V\rangle .
\end{aligned}
$$

Since at $z_{0}$,

$$
\langle U-A, V\rangle=\frac{1}{2} \frac{d}{d z}\|U-A\|^{2}>0
$$

we conclude that $\langle U-A, V\rangle$ is increasing. Therefore, $\langle U-A, V\rangle>0$ for $z>z_{0}$ as long as $U(z) \in X_{2}$. Finally, if $z>z_{0}$ and $U(z) \in X_{2}$, then

$$
\frac{d}{d z}\|U(z)-A\|^{2}=2\langle U-A, V\rangle>0
$$

To complete the proof of Lemma 4A.1 we observe that our proof that $I$ is open also implies that $\varphi(\theta, q)$ can be chosen to depend continuously on $\theta$ and $q$.

\section{REFERENCES}

1. C. Conley, Isolated invariant sets and the Morse index, CBMS Regional Conf. Ser. in Math., no. 38, Amer. Math. Soc., Providence, R. I., 1978.

2. P. Hartman, Ordinary differential equations, 2nd ed, Birkhauser, Boston, Mass., 1982.

3. D. Terman, Directed graphs and traveling waves, Trans. Amer. Math. Soc. 289 (1985), 809-847.

4. Infinitely many radial solutions of an elliptic system (submitted).

Department of Mathematics, Michigan State University, East Lansing, Michigan 48824

Current address: Department of Mathematics, Ohio State University, Columbus, Ohio 43210 